\title{
A second order method for solving turbulent shallow flows
}

\author{
J. Fe \& F. Navarrina \\ Grupo de Métodos Numéricos en Ingeniería, \\ University of A Coruña, Spain
}

\begin{abstract}
A second order finite volume model for the resolution of the two dimensional shallow water equations with turbulent term is presented. It is shown that, if a first order upwind method is used to discretize the hydrodynamic equations, a considerable amount of numerical viscosity (or diffusion) is produced. For this reason a second order method has been developed, which makes use of the mean gradient of the variables in a cell. To compare the first and second order methods, the Cavity Flow problem is used. Then a backward step problem is solved, using the $k-\varepsilon$ turbulence model to calculate the turbulent viscosity at every point. The results are compared with experimental measures and they confirm the good behavior of the model.
\end{abstract}

Keywords: finite volumes, shallow water equations, numerical viscosity, turbulent term, gradient mean values.

\section{Introduction}

The two-dimensional shallow water equations (2D-SWE) describe the behavior of free surface flows in which the ratio of the depth to the horizontal dimensions is small and the magnitude of the vertical velocity component is much smaller than the magnitude of the horizontal velocity components. This situation can be found for instance in the flow in channels and rivers.

2D-SWE take into account the turbulence effects both through the frictional terms and the second derivatives term. This last term may not be significant in many practical problems when we only need an estimate of energy losses. However, its inclusion may become very important for an accurate simulation of recirculating flows. 
In a first approach we discretized the hydrodynamic equations with a first order finite volume method. An important point when working with this method is to properly calculate the numerical flux at the cell edges. The upwinding of the flux term has proved to be a useful technique, but it produces a considerable amount of numerical diffusion. For this reason it has been developped a second order method that makes use of the mean gradient of the velocity components in a cell.

To compare the first and second order methods for uniform viscosity values, the Cavity Flow problem has been used with three viscosity values. Then a Backward Step problem has been solved and its results have been compared with experimental measures. The eddy viscosity values at every point have been generated with the depth averaged $k-\varepsilon$ model.

In this work: 1) we describe the first and second hydrodynamic models; 2) we compare them in the Cavity Flow problem; 3) we combine the second order hydrodynamic model with the first order $k-\varepsilon$ turbulence model, comparing the obtained results with experimental measures.

\section{The shallow water equations}

The 2D-SWE system in conservative form is expressed as

$$
\frac{\partial \mathbf{U}}{\partial t}+\frac{\partial \mathbf{F}_{\mathbf{1}}}{\partial x}+\frac{\partial \mathbf{F}_{\mathbf{2}}}{\partial y}=\mathbf{G}
$$

being the vector of unknowns $\mathbf{U}$ and the flux terms

$$
\mathbf{U}=\left(\begin{array}{c}
h \\
h u \\
h v
\end{array}\right), \quad \mathbf{F}_{\mathbf{1}}=\left(\begin{array}{c}
h u \\
h u^{2}+\frac{1}{2} g h^{2} \\
h u v
\end{array}\right), \quad \mathbf{F}_{\mathbf{2}}=\left(\begin{array}{c}
h v \\
h u v \\
h v^{2}+\frac{1}{2} g h^{2}
\end{array}\right)
$$

and being the source term

$$
\mathbf{G}=\left(\begin{array}{c}
0 \\
g h\left(S_{0 x}-S_{f x}\right)+S_{t 1} \\
g h\left(S_{0 y}-S_{f y}\right)+S_{t 2}
\end{array}\right)
$$

In the above expressions $h$ is the fluid depth, $u$ and $v$ are the horizontal velocity components and $g$ is the gravity acceleration. $S_{0 x}, S_{0 y}$ are the geometric slopes. $S_{f x}, S_{f y}$ are the friction slopes

$$
S_{f x}=\frac{n^{2} u \sqrt{u^{2}+v^{2}}}{R_{h}^{4 / 3}}, \quad S_{f y}=\frac{n^{2} v \sqrt{u^{2}+v^{2}}}{R_{h}^{4 / 3}},
$$


where $R_{h}$ is the hydraulic radius. Finally, $S_{t 1}, S_{t 2}$ are the turbulent terms

$$
\begin{aligned}
& S_{t 1}=\frac{\partial}{\partial x}\left(2 \nu_{t} h \frac{\partial u}{\partial x}\right)+\frac{\partial}{\partial y}\left(\nu_{t} h\left[\frac{\partial v}{\partial x}+\frac{\partial u}{\partial y}\right]\right), \\
& S_{t 2}=\frac{\partial}{\partial x}\left(\nu_{t} h\left[\frac{\partial v}{\partial x}+\frac{\partial u}{\partial y}\right]\right)+\frac{\partial}{\partial y}\left(2 \nu_{t} h \frac{\partial v}{\partial y}\right),
\end{aligned}
$$

where the coefficient $\nu_{t}$ is a variable called eddy (or turbulent) viscosity.

\section{Discretization of the equations}

\subsection{Construction of the finite volume mesh}

The finite volumes used in this work are based on a triangular discretization of the domain (see Figure 1). For each node I, the barycenters of all the triangles that have the common vertex $\mathrm{I}$ as well as the midpoints of the corresponding edges are considered. The boundary $\Gamma_{i}$ of the cell $\mathrm{C}_{i}$ is defined by these points. By $\Gamma_{i j}=\overline{\mathrm{AMB}}$ we represent the part of $\Gamma_{i}$ that is also part of $\Gamma_{j}$. The outward normal vector to $\Gamma_{i j}$ is $\boldsymbol{\eta}_{i j}$. The norm of $\boldsymbol{\eta}_{i j},\left\|\boldsymbol{\eta}_{i j}\right\|$, is the length of the edge and $\widetilde{\boldsymbol{\eta}}_{i j}=\left(\widetilde{\alpha}_{i j}, \widetilde{\beta}_{i j}\right)^{T}$ is the corresponding unit vector. The subcell $\mathrm{T}_{i j}$ is the union of triangles AMI and MBI.

\subsection{Discretization of the hydrodynamic equations}

At this point we wish to integrate the 2D-SWE, what results in

$$
\iint_{\mathrm{C}_{i}} \frac{\partial \mathbf{U}}{\partial t} d A+\iint_{\mathrm{C}_{i}} \boldsymbol{\nabla} \cdot \mathcal{F} d A=\iint_{\mathrm{C}_{i}} \mathbf{G} d A,
$$

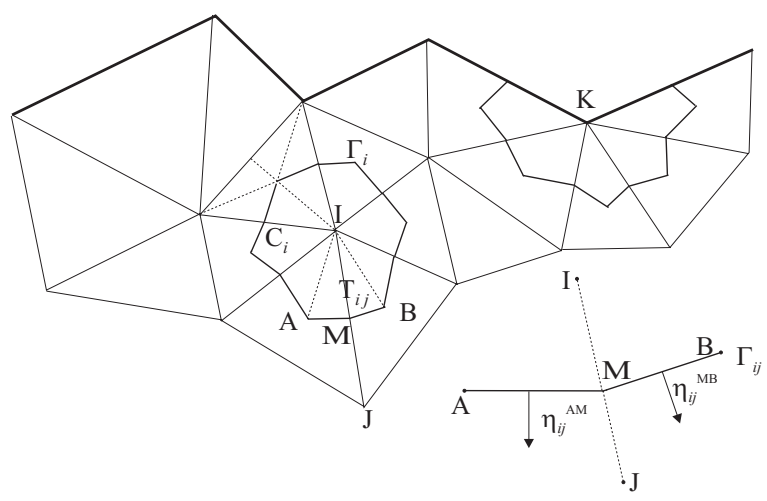

Figure 1: Finite volumes construction. 
where the operator $\nabla$ stands for $\left(\frac{\partial}{\partial x}, \frac{\partial}{\partial y}\right)$ and $\mathcal{F}=\left(\mathbf{F}_{\mathbf{1}}, \mathbf{F}_{\mathbf{2}}\right)$. If we apply the Gauss theorem to the flux term, it results

$$
\iint_{\mathrm{C}_{i}} \frac{\partial \mathbf{U}}{\partial t} d A+\int_{\Gamma_{i}} \mathcal{F} \cdot \tilde{\boldsymbol{\eta}} d A=\iint_{\mathrm{C}_{i}} \mathbf{G} d A
$$

The details on the application of the FVM to the 2D-SWE, by making use of the upwind Van Leer Q-scheme [1], can be found in Fe et al. [2]. The discretized expresion of the 2D-SWE that corresponds to node I is

$$
\frac{\mathbf{U}_{i}^{n+1}-\mathbf{U}_{i}^{n}}{\Delta t} A_{i}+\sum_{j \in \mathcal{K}_{i}}\left\|\boldsymbol{\eta}_{i j}\right\| \boldsymbol{\phi}_{i j}^{n}=\sum_{j \in \mathcal{K}_{i}}\left(A_{i j} \boldsymbol{\psi}_{i j}^{n}+\left\|\boldsymbol{\eta}_{i j}\right\| \boldsymbol{\psi}_{\nu i j}^{n}\right)
$$

in which $\mathbf{U}_{i}^{n}$ and $\mathbf{U}_{i}^{n+1}$ are approximations to the solution of eqn (1) within each cell $\mathrm{C}_{i}$ and at time steps $t_{n}$ and $t_{n+1} . A_{i}$ and $A_{i j}$ are the cell and subcell areas. $\mathcal{K}_{i}$ represents the set of neighboring nodes of I. The numerical flux $\boldsymbol{\phi}_{i j}^{n}$ is the approximation of $\mathbf{Z}=\mathcal{F} \cdot \widetilde{\boldsymbol{\eta}}$, at $\Gamma_{i j}, j \in \mathcal{K}_{i}$ and at $t=t_{n}$, and it is given by

$$
\boldsymbol{\phi}_{i j}^{n}=\frac{\mathbf{Z}\left(\mathbf{U}_{i}^{n}, \widetilde{\boldsymbol{\eta}}_{i j}\right)+\mathbf{Z}\left(\mathbf{U}_{j}^{n}, \widetilde{\boldsymbol{\eta}}_{i j}\right)}{2}-\frac{1}{2}\left|\mathbf{Q}\left(\mathbf{U}_{Q}^{n}, \widetilde{\boldsymbol{\eta}}_{i j}\right)\right|\left(\mathbf{U}_{j}^{n}-\mathbf{U}_{i}^{n}\right) .
$$

$\mathbf{Q}$ is the jacobian matrix of $\mathbf{Z} .|\mathbf{Q}|$ is defined as $\mathbf{X}|\boldsymbol{\Lambda}| \mathbf{X}^{-1}$, where $|\boldsymbol{\Lambda}|$ is the diagonal matrix given by the absolute values of the eigenvalues of $\mathbf{Q}$ and $\mathbf{X}$ is the eigenvectors matrix of $\mathbf{Q}$. $\mathbf{U}_{Q}$ represents the vector of variables at the midpoint between I and J.

The numerical source in eqn (9) has two terms. In the first of them, it is calculated as

$$
\boldsymbol{\psi}_{i j}^{n}=\left(\mathbf{I}-|\mathbf{Q}| \mathbf{Q}^{-1}\right) \widehat{\mathbf{G}}_{\mathbf{0}}+\widehat{\mathbf{G}}_{\mathbf{f}}
$$

where the numerical geometric and friction slopes are respectively

$$
\widehat{\mathbf{G}}_{\mathbf{0}}=\left(\begin{array}{c}
0 \\
g \frac{h_{i}^{n}+h_{j}^{n}}{2} \frac{H_{j}-H_{i}}{d_{i j}} \widetilde{\alpha} \\
g \frac{h_{i}^{n}+h_{j}^{n}}{2} \frac{H_{j}-H_{i}}{d_{i j}} \widetilde{\beta}
\end{array}\right), \quad \widehat{\mathbf{G}}_{\mathbf{f}}=\left(\begin{array}{c}
0 \\
g h_{i}^{n}\left(-S_{f x}\right)_{i}^{n} \\
g h_{i}^{n}\left(-S_{f y}\right)_{i}^{n}
\end{array}\right),
$$

being $d_{i j}$ the normal distance from I to $\Gamma_{i j}$. It can be noted that the numerical geometric slope $\widehat{\mathbf{G}}_{0}$ is upwinded [1], while the numerical friction slope $\widehat{\mathbf{G}}_{\mathrm{f}}$ is 
discretized pointwise [3], which is a widespread method to treat this term. In the second term, the numerical source takes the form $\boldsymbol{\psi}_{\nu i j}^{n}=\widehat{\mathrm{G}}_{\mathbf{t}}$, being

$$
\widehat{\mathbf{G}}_{\mathbf{t}}=\left\{\begin{array}{c}
0 \\
\frac{\nu_{t i}+\nu_{t j}}{2} \frac{h_{i}^{n}+h_{j}^{n}}{2}\left(2 \frac{u_{x i}^{n}+u_{x j}^{n}}{2} \widetilde{\alpha}+\frac{v_{x i}^{n}+v_{x j}^{n}}{2} \widetilde{\beta}+\frac{u_{y i}^{n}+u_{y j}^{n}}{2} \widetilde{\beta}\right) \\
\frac{\nu_{t i}+\nu_{t j}}{2} \frac{h_{i}^{n}+h_{j}^{n}}{2}\left(\frac{v_{x i}^{n}+v_{x j}^{n}}{2} \widetilde{\alpha}+\frac{u_{y i}^{n}+u_{y j}^{n}}{2} \widetilde{\alpha}+2 \frac{v_{y i}^{n}+v_{y j}^{n}}{2} \widetilde{\beta}\right)
\end{array}\right\}
$$

the numerical turbulent slope. The eddy viscosities $\nu_{t i}$ and $\nu_{t j}$ have no time indices since it is assumed that they are constant at each node throughout the hydrodynamic computational process. The values $u_{x i}^{n}, u_{y i}^{n}, v_{x i}^{n}, v_{y i}^{n}$ represent the average of the derivatives of $u, v$ at cell $\mathrm{C}_{i}$ and at time $t=t_{n}$.

$$
\begin{aligned}
u_{x i}^{n} & =\left(\overline{\frac{\partial u}{\partial x}}\right)_{\mathrm{C}_{i}, t_{n}}, \quad u_{y i}^{n}=\left(\overline{\frac{\partial u}{\partial y}}\right)_{\mathrm{C}_{i}, t_{n}}, \\
v_{x i}^{n} & =\left(\overline{\frac{\partial v}{\partial x}}\right)_{\mathrm{C}_{i}, t_{n}}, \quad v_{y i}^{n}=\left(\overline{\frac{\partial v}{\partial y}}\right)_{\mathrm{C}_{i}, t_{n}} .
\end{aligned}
$$

These averaged values can be calculated from the values at the cell edges and a way to calculate them is shown et al. [2].

Equation (9) provides then a time explicit method to calculate the variables, at every node I and at every time step, from the previous time step values at node I and its neighboring nodes.

\section{The second order model}

\subsection{The cavity flow test: first results}

Now we are going to test the first order model by using the Cavity flow problem, a classical benchmark for the two dimensional Navier-Stokes equations (2D-NSE). 2D-SWE are obtained from the three dimensional Navier-Stokes equations and they are different from the 2D-NSE. The latter do not take into account the third dimension in space, but only the velocities and pressures of a theoretical planar flow, whereas 2D-SWE consider the third dimension by means of the variable depth, and the pressure is expressed as a function of the depth. However both systems produce very similar results with uniform viscosity values and the results of this test may be very useful to asses the ability of the proposed model to accurately represent viscous flows.

The problem consists in obtaining the velocity field in a square domain of $1 \times$ $1 \mathrm{~m}^{2}$. A regular mesh of $81 \times 81$ nodes is employed. The boundary conditions, of 

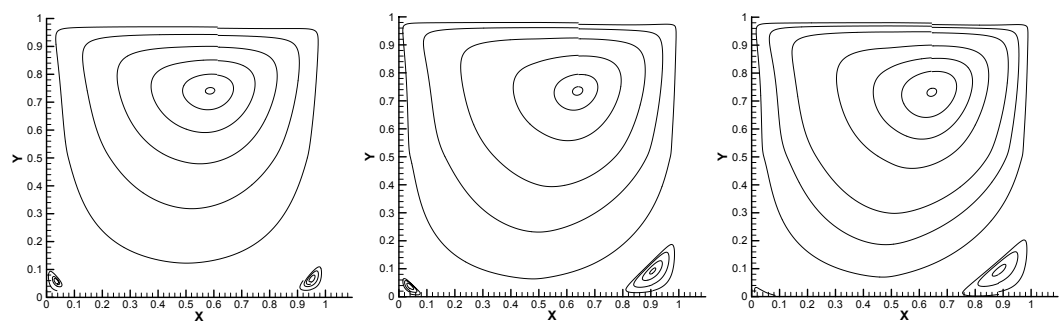

Figure 2: First order model: a) $\nu=0.01$; b) $\nu=0.001$; c) $\nu=0.0001$.

Dirichlet type, are: $u=1, v=0$ at the upper side; $u=v=0$ (no-slip condition) at the other three. In the Cavity Flow problem, the form of the streamlines depend on the flow Reynolds number $R e$. Taking the values $V=1$ and $L=1$ for the velocity and length scales, the resulting $R e$ is

$$
R e=\frac{V L}{\nu}=\frac{1}{\nu},
$$

which allows us to simulate Reynolds numbers of 100, 1000, 10000 by varying the viscosity value. The resulting streamlines are shown in Figure 2 and it can be observed that the difference between the three cases is much smaller than what should be expected with a difference in viscosity values of almost $0.01 \mathrm{~m}^{2} / \mathrm{s}$. The reason must be found in the high numerical viscosity (or diffusion) inherent in first order upwind methods. This numerical viscosity is known to depend on the mesh size and, even with a reasonably fine mesh such as the one used here, its effects are unacceptable. A method to reduce it with a first order method has already been proposed [2], but it reduces the stability as well. For this reason we have developed a second order model.

\subsection{The mean gradient}

An important aspect, when working with the finite volume method, is to properly calculate the numerical flux at the cell edges. In the first order method described before, it has been supposed that the values of $u, v$ were uniform within each of the two cells $C_{i}$ and $C_{j}$ having a common interface $\Gamma_{i j}$. The main point to obtain a second order model is to employ instead a linear approximation for the variables within each cell. To this end we have used the mean gradient of the velocity components, which was calculated in Section 3. This mean gradient is obtained from the values at the cell edges, thus involving the variables values at the adjacent cells.

Let us take, for instance, the variable $u$ and call $u_{i}, u_{j}$ its uniform values within $C_{i}$ and $C_{j}$. The linear reconstruction produces, at each side of $\Gamma_{i j}$, the following values of $u$ : - Side $C_{i}: u_{i}^{*}=u_{i}+(\overline{\nabla u})_{C_{i}} \cdot\left(\mathbf{r}_{M}-\mathbf{r}_{I}\right)$.

- Side $C_{j}: u_{j}^{*}=u_{j}+(\overline{\nabla u})_{C_{j}} \cdot\left(\mathbf{r}_{M}-\mathbf{r}_{J}\right)$, 


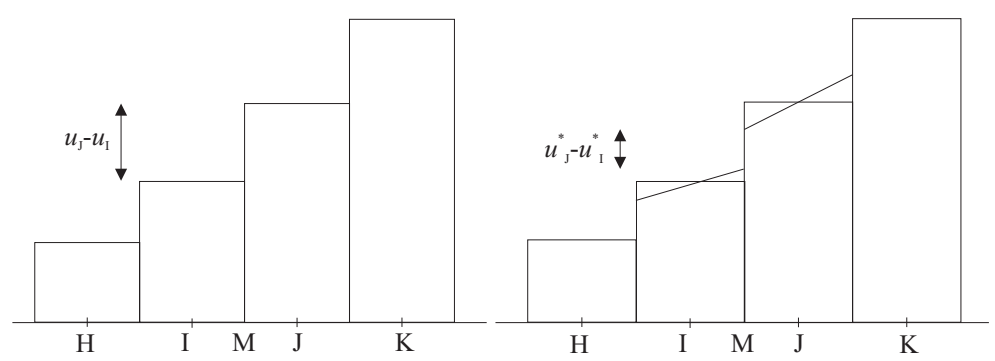

Figure 3: a) First order scheme, b) Second order scheme.
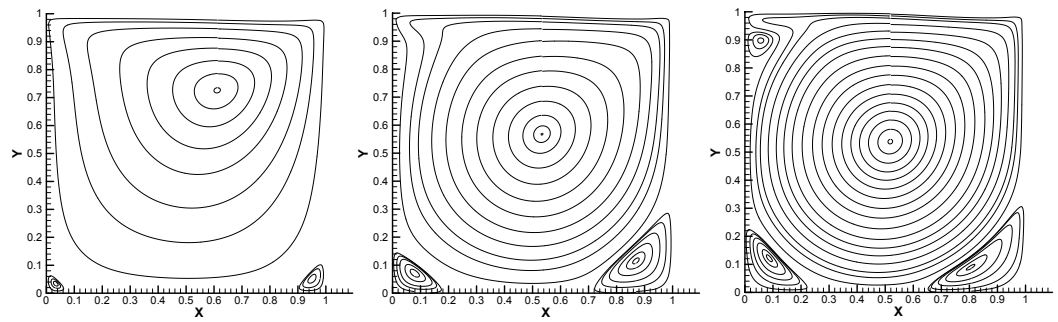

Figure 4: Second order model: a) $\nu=0.01$; b) $\nu=0.001$; c) $\nu=0.0001$.
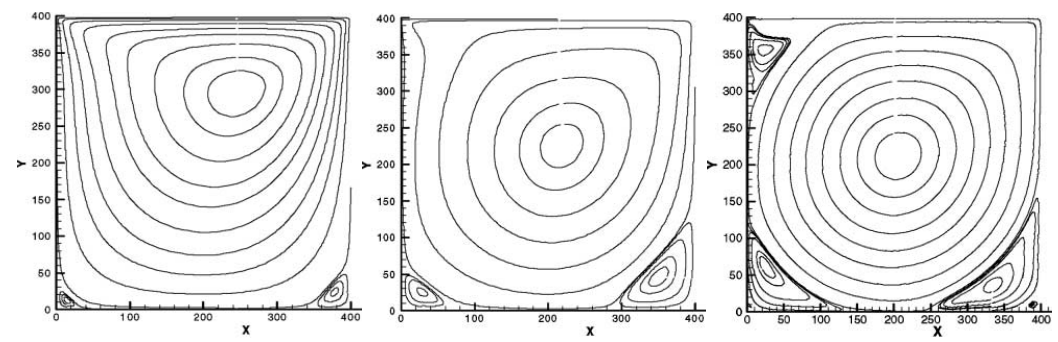

Figure 5: Reference streamlines: a) $\nu=0.01$; b) $\nu=0.001$; c) $\nu=0.0001$.

being $M \in \Gamma_{i j}$ the midpoint between $I$ and $J$. In this way the difference between the $u$ values on both sides of the interface, which is responsible for the numerical viscosity added, is reduced (see Figure 3). The streamlines obtained using the second order scheme are shown in Figure 4 and we see that they agree very well with the reference streamlines taken from Vellando et al. [4] (Figure 5).

\subsection{The turbulence model}

As it has been shown, the 2D study of viscous fluids with Reynolds numbers below 10.000 can be carried out by using constant values for the viscosity. To represent real turbulent flows, however, it is necessary to calculate the turbulent viscosity $\nu_{t}$ at every point. To obtain it we have used the depth-averaged $k-\varepsilon$ turbulence 
model [5] where $\nu_{t}$ is calculated as

$$
\nu_{t}=c_{\mu} \frac{k^{2}}{\varepsilon},
$$

being $k$ and $\varepsilon$ the turbulence kinetic energy and the dissipation rate per unit mass respectively. They are given by the transport equations

$$
\begin{gathered}
\frac{\partial k}{\partial t}+u \frac{\partial k}{\partial x}+v \frac{\partial k}{\partial y}=\frac{\partial}{\partial x}\left(\frac{\nu_{t}}{\sigma_{k}} \frac{\partial k}{\partial x}\right)+\frac{\partial}{\partial y}\left(\frac{\nu_{t}}{\sigma_{k}} \frac{\partial k}{\partial y}\right)+P_{h}+P_{k V}-\varepsilon, \\
\frac{\partial \varepsilon}{\partial t}+u \frac{\partial \varepsilon}{\partial x}+v \frac{\partial \varepsilon}{\partial y}=\frac{\partial}{\partial x}\left(\frac{\nu_{t}}{\sigma_{\varepsilon}} \frac{\partial \varepsilon}{\partial x}\right)+\frac{\partial}{\partial y}\left(\frac{\nu_{t}}{\sigma_{\varepsilon}} \frac{\partial \varepsilon}{\partial y}\right)+c_{1 \varepsilon} \frac{\varepsilon}{k} P_{h}+P_{\varepsilon V}-c_{2 \varepsilon} \frac{\varepsilon^{2}}{k} .
\end{gathered}
$$

A way to implement these equations together with the hydrodynamic equations has been fully described in Fe et al. [6]).

\subsection{Measurement of the velocity and turbulent kinetic energy}

The model has been applied to a real channel and experimental measurements of the velocity components have also been made, from which we have calculated the turbulent kinetic energy $k$. The experimental data were obtained at the Hydraulics Laboratory of the Civil Engineering School of A Coruña with SONTEK Micro Acoustic Doppler Velocimeters that produce a small distortion of the velocity field. They are highly accurate $\left(10^{-3} \mathrm{~m} / \mathrm{s}\right)$ and they take between 80 and 250 measurements per second. As the output values have a maximum frequency of $50 \mathrm{~Hz}$, every one represents an average of several measurements. In our case, 2500 values of the velocity components were obtained at every point, during a period of $100 \mathrm{~s}$. The velocimeters were placed at a distance from the bottom of $9.36 \mathrm{~cm}$, at 368 points.

The system gives the three mean velocities $u, v, w$ and the three standard deviations $\sigma_{x}, \sigma_{y}, \sigma_{z}$. Since the model is two-dimensional only the $x$ and $y$ deviation have been taken into account, what seems more coherent with the previous hypothesis. The experimental value of the turbulent kinetic energy is then calculated as

$$
k=\frac{1}{2}\left({\sigma_{x}}^{2}+{\sigma_{y}}^{2}\right) .
$$

\subsection{Description of the installation and boundary conditions}

The experimental domain consisted of a horizontal channel made of glass with an abrupt expansion, commonly known as Backward Step. The dimensions can be seen in Figure 6. For the experimental process, a discharge of $Q=20.2 \mathrm{l} / \mathrm{s}$, was employed and a depth of $h=24.2 \mathrm{~cm}$ was measured at the end of the channel. Both were the upstream and downstream boundary conditions used for the numerical model. At the walls, the friction velocity condition, described in Fe et al. [6], was considered. 


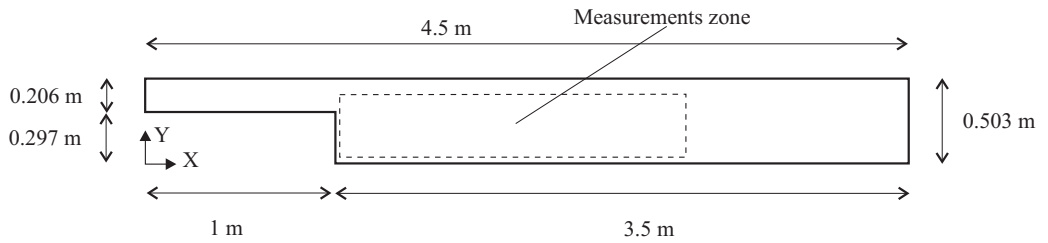

Figure 6: Dimensions of the domain.

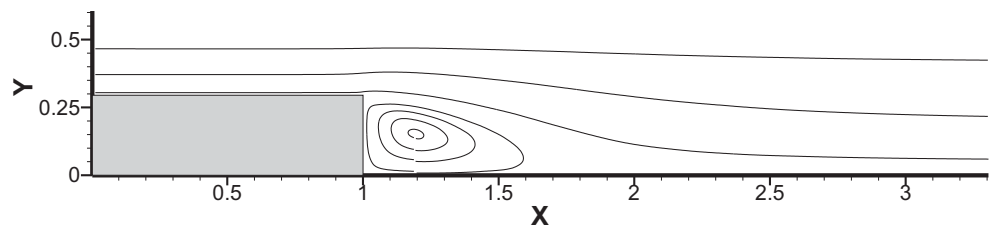

Figure 7: First order model. Streamlines. General view.

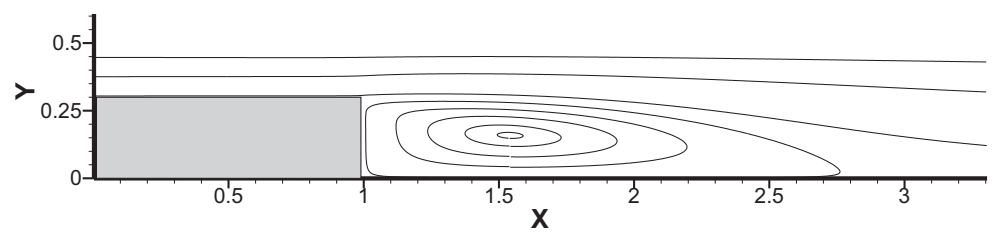

Figure 8: Second order model. Streamlines. General view.

\subsection{Results}

The model was applied with a first order discretization. We noticed that the influence of using the $k-\varepsilon$ model to generate the $\nu_{t}$ values was insignificant, due again to the numerical viscosity introduced by the upwinding. The results show a reattachment length too short (Figure 7).

Then the second order model was used. The resulting streamlines simulate much better the ones obtained from the experimental measures. They are presented in Figure 8 in a general view, and in Figure 9 in an enlarged view to compare them with the experimental results (Figure 10). The computational results for $k$ are shown in Figure 11. The levels are well predicted, but the position is not so accurately assessed. Due to the simplifying hypothesis made for the walls, the model fails to reproduce the high $k$ levels near the right wall of the channel.

\section{Conclusions}

We have shown that the consideration of the turbulent term improves the accuracy in the representation of $2 \mathrm{D}$ recirculating viscous flows, provided that the numerical viscosity produced by the upwinding is reduced. To this end a second order dis- 


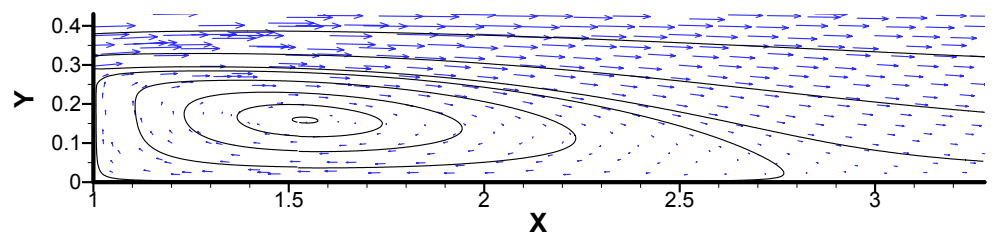

Figure 9: Second order model. Streamlines and velocity vectors.

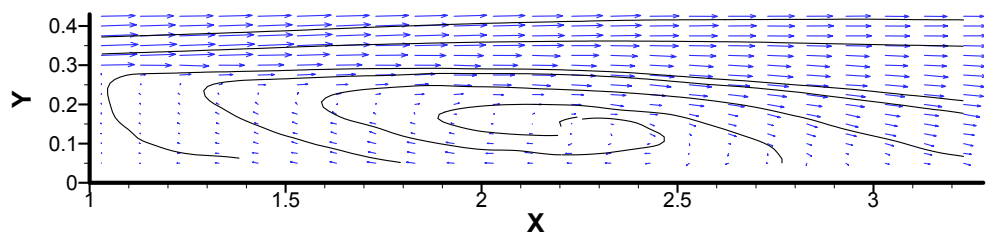

Figure 10: Experimental measures. Streamlines and velocity vectors.

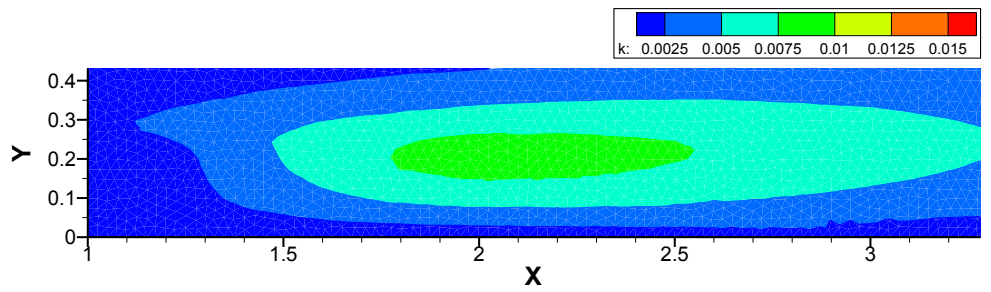

Figure 11: Second order model. Turbulent kinetic energy.

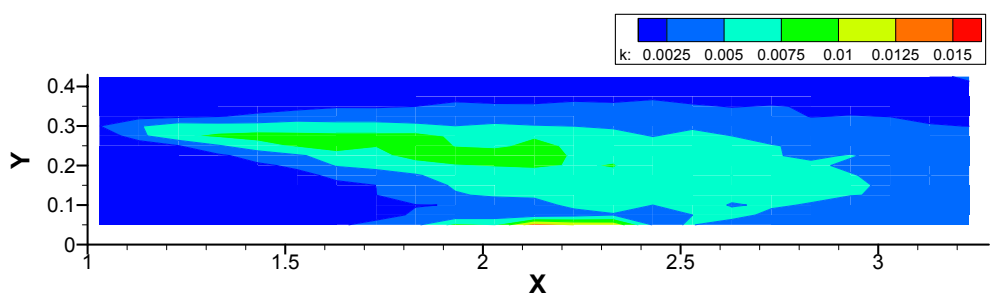

Figure 12: Experimental measures. Turbulent kinetic energy.

cretization of the velocity components has been proposed, achieving an accurate resolution of the velocity field in the three Cavity Flow tests solved.

The second order model, in combination with a $k-\varepsilon$ model, has produced a remarkable improvement with respect to the first order model in a Backward Step problem. The proposed model has correctly calculated the position and length of the eddy. The computed $k$ levels are close to the ones obtained from experimental measures. 


\section{Acknowledgements}

This work has been partially supported by Grants \# DXPCTSU-CEOU-2007/009 and PGDIT06TAM 118001PR of the Xunta de Galicia and by research fellowships of the Universidad de A Coruña and the Fundación de la Ingeniería Civil de Galicia.

\section{References}

[1] Bermúdez, A., Dervieux, A., Desideri, J. \& Vázquez, M.E, Upwind schemes for the two dimensional shallow water equations with variable depth using unstructured meshes. Comput. Methods Appl. Mech. Eng., 155, pp. 49-72, 1998.

[2] Fe, J., Cueto-Felgueroso, L., Navarrina, F. \& Puertas, J., Numerical viscosity reduction in the resolution of the shallow water equations with turbulent term. International Journal for Numerical Methods in Fluids 2008; 58, pp. 781-802.

[3] Brufau, P., Vázquez-Cendón, M.E. \& García-Navarro, P., A numerical model for the flooding and drying of irregular domains. International Journal for Numerical Methods in Fluids 2002, 39, pp. 247-275.

[4] Vellando, P., Puertas, J. \& Colominas, I. SUPG stabilized finite element resolution of the Navier-Stokes equations. Applications to water treatment engineering. Computer Methods in Applied Mechanics and Engineering 2002; 191, pp. 5899-5922.

[5] Rodi, W., Turbulence models and their application in hydraulics. A state-ofthe-art review, IAHR monograph, Balkema: Rotterdam, 1993.

[6] Fe, J., Navarrina, F., Puertas, J., Vellando, P., Ruiz, D., Experimental validation of two depth-averaged turbulence models, International Journal for Numerical Methods in Fluids; published on-line in Wiley InterScience (www.interscience.wiley.com), DOI: 10.1002/fld.1880. 